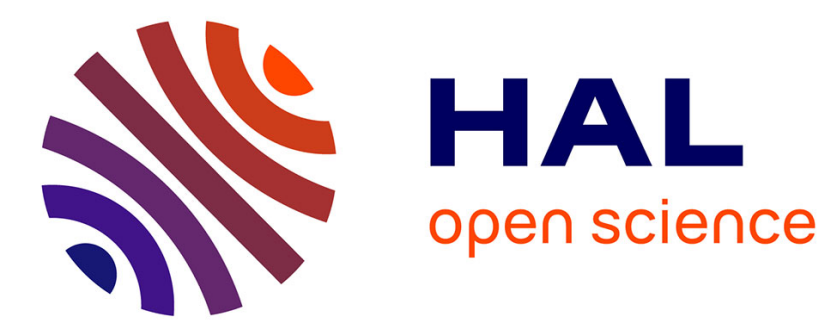

\title{
Amoeboid Swimming: A Generic Self-Propulsion of Cells in Fluids by Means of Membrane Deformations
}

\author{
Alexander Farutin, Salima Rafaï, Dag Kristian Dysthe, Alain Duperray,
} Philippe Peyla, Chaouqi Misbah

\section{- To cite this version:}

Alexander Farutin, Salima Rafaï, Dag Kristian Dysthe, Alain Duperray, Philippe Peyla, et al.. Amoeboid Swimming: A Generic Self-Propulsion of Cells in Fluids by Means of Membrane Deformations. Physical Review Letters, 2013, 111 (22), pp.228102. 10.1103/PhysRevLett.111.228102 . hal-00908796

\section{HAL Id: hal-00908796 \\ https://hal.science/hal-00908796}

Submitted on 25 Nov 2013

HAL is a multi-disciplinary open access archive for the deposit and dissemination of scientific research documents, whether they are published or not. The documents may come from teaching and research institutions in France or abroad, or from public or private research centers.
L'archive ouverte pluridisciplinaire HAL, est destinée au dépôt et à la diffusion de documents scientifiques de niveau recherche, publiés ou non, émanant des établissements d'enseignement et de recherche français ou étrangers, des laboratoires publics ou privés. 


\title{
Amoeboid Swimming: A Generic Self-Propulsion of Cells in Fluids by Means of Membrane Deformations
}

\author{
Alexander Farutin, ${ }^{1, *}$ Salima Rafaï, ${ }^{1}$ Dag Kristian Dysthe, ${ }^{2}$ \\ Alain Duperray, ${ }^{3}$ Philippe Peyla, ${ }^{1}$ and Chaouqi Misbah ${ }^{1, \dagger}$ \\ ${ }^{1}$ Laboratory of Interdisciplinary Physics, UMR 5588, \\ Université Joseph Fourier and CNRS, F-38041 Grenoble, France \\ ${ }^{2}$ University of Oslo, Physics of Geological Processes, P.O. Box 1048 Blindern, N-0316 Oslo, Norway \\ ${ }^{3}$ Centre de Recherche INSERM U823 Institut Albert Bonniot BP170 38042 Grenoble Cedex 9 France
}

(Dated: Received: November 25, 2013/ Revised version: (date))

\begin{abstract}
Microorganisms, such as bacteria, algae, or spermatozoa, are able to propel themselves forward thanks to flagella or cilia activity. By contrast, other organisms employ pronounced changes of the membrane shape to achieve propulsion, a prototypical example being the Eutreptiella gymnastica. Cells of the immune system as well as dictyostelium amoebae, traditionally believed to crawl on a substratum, can also swim in a similar way. We develop a model for these organisms: the swimmer is mimicked by a closed incompressible membrane with force density distribution (with zero total force and torque). It is shown that fast propulsion can be achieved with adequate shape adaptations. This swimming is found to consist of an entangled pusher-puller (EPP) state. The auto-propulsion distance over one cycle is a universal linear function of a simple geometrical dimensionless quantity $A / V^{2 / 3}$ ( $V$ and $A$ are cell volume and its membrane area). This study captures the peculiar motion of Eutreptiella gymnastica with simple force distribution.
\end{abstract}

PACS numbers: 47.63.Gd, 47.15.G-, 47.61.-k

Introduction In nature, organisms that can propel themselves in a fluid medium are ubiquitous. While larger organisms, such as fish, use inertia in their motion, some micro-organisms move at low Reynolds number $(R e)$, where viscous forces dominate inertial effects: They are classified as micro-swimmers [1, 2]. Among them, a lot of micro-organisms [3, 4], like spermatozoa [5], bacteria [6], or micro-algae [7, 8], can move with the help of flagella or cilia $[6,9]$ and a very important literature has been recently dedicated to them in order to understand the complex coupling between their motion and the hydrodynamics of the surrounding fluid.

However, some other organisms, like Eutreptiella gymnastica [10] (a common representative of euglenids of marine phytoplankton), use both flagella and pronounced shape changes to swim. While the conventional mode for amoeboid locomotion is crawling on solid surfaces, it was shown recently $[11,12]$ that dictyostelium amoebae are also able to swim by deforming their body with a process similar to the one they use for crawling. Indeed, they can swim with speeds similar to those on a solid substrate. A similar behavior [11] is observed for neutrophils, i.e. the white blood cells in mammals that form an essential part of the innate immune system.

This peculiar self-propulsion with shape deformations requires the movement of the cell's surface to occur from the cell's front toward its rear and this deformation moves back and forth along the swimming direction. Because of the purcell's theorem [1] this motion at low Re must break the reciprocal time symmetry.

Theoretical studies of self-propulsion with shape deformations have been considered for ellipsoidal particles
[13, 14], and general strokes[15-18] or shapes[19] optimizing the dissipation. The physics of a cell membrane incompressibility adopted here (verified for eutreptiella $[20]$ ), will be seen to have a strong impact on swimming. On the one hand, local inextensibility of the cell membrane is a constraint that is known to induce a variety of dynamical motions (like within a red blood cell) [21] which are absent for droplets. On the other hand, membrane incompressibility limits membrane protrusions in the motility process $[11,12]$. This is reflected on the propulsion law, as we shall see.

We propose an elementary model describing the selfpropulsion of a cell due to shape deformation of its inextensible membrane. We first consider a quasi-spherical swimmer for which an explicit analytical solution can be obtained. We then explore large swimmer deformations by means of numerical simulations. This minimal model captures several swimming features. We make predictions that are not devoid of experimental testability.

The model The swimmer has inextensible fluid membrane encapsulating a newtonian fluid with viscosity $\lambda \eta$ and floating in fluid of viscosity $\eta$ ( $\lambda$ is viscosity contrast). The swimmer is capable of changing its shape by applying active forces from its membrane. We restrict the model to normal active forces, in order to remain within a minimal model, although tangential active forces can be accounted for in principle. Due to inextensibility the membrane exerts passive tension forces on the surrounding liquids in reaction to active forces. We assume that there is no exchange of matter between the interior and exterior of the swimmer on the relevant time scales, as well as no-slip boundary conditions at the membrane. Fi- 
nally, the swimmer is considered as neutrally buoyant, so that the total force and torque exerted by the swimmer on the liquids vanish at each time. Our model can be viewed in a first step applicable to artificial swimmers, made of vesicles or red blood cells. This work should serve as a basis for more refined studies relevant to complex biological cells.

Under these conditions, the state of a microswimmer is fully described by its shape and position and the only conserved geometrical properties are the volume $V$ and the surface area $A$ (analysis of some specimens of euglenid swimming supports these assumptions [20]).

Let $r_{v}$ be the radius of the equivalent sphere, such that $V=4 \pi r_{v}^{3} / 3$. The deflation from the sphere is specified by the normed excess area $\Gamma, A=4 \pi r_{v}^{2}(1+\Gamma)(\Gamma=0$ for a sphere).

Analytical results can be obtained for an almostspherical swimmer $(\Gamma<<1)$. The deviation from a sphere $\left(r=r_{v}\right)$ of the shape of the swimmer is parameterized (in the comoving frame) by the scalar function:

$$
\rho(\boldsymbol{x})=\sum_{l=0}^{l_{\max }} \sum_{m=-l}^{l} \rho_{l, m} Y_{l, m}(\boldsymbol{x})
$$

where $Y_{l, m}$ are spherical harmonics, $\boldsymbol{x} \equiv \boldsymbol{r} / r, \boldsymbol{r}$ represents swimmer membrane vector position, and $\rho_{l, m}$ are time-dependent amplitudes (to be determined). $l_{\max }$ specifies the maximum number of harmonics. We have checked that using harmonics of order higher than 6 does not result in a noticeable increase of traveled distance during one stroke cycle.

Likewise, the amplitude of the active forces $F(\boldsymbol{x})$ exerted by the swimmer on the fluid is decomposed as in (1) with amplitudes $F_{l, m}$. To enforce local membrane incompressibility a Lagrange multiplier $\zeta(\boldsymbol{x})$ is introduced, which acts as a position-dependent surface tension. The corresponding force takes the form (see [22]).

$$
\boldsymbol{F}^{\text {tens }}(\boldsymbol{x})=-H(\boldsymbol{x}) \zeta(\boldsymbol{x}) \boldsymbol{n}(\boldsymbol{x})+\boldsymbol{\nabla}^{s} \zeta(\boldsymbol{x}),
$$

where $H(\boldsymbol{x})$ is the mean curvature, $\boldsymbol{n}(\boldsymbol{x})$ is the outward normal to the membrane, and $\boldsymbol{\nabla}^{s}=[\boldsymbol{I}-\boldsymbol{n}(\boldsymbol{x}) \otimes \boldsymbol{n}(\boldsymbol{x})] . \boldsymbol{\nabla}$ is the surface gradient operator on the membrane. The local incompressibility of the membrane demands that the velocity field $\boldsymbol{v}(\boldsymbol{x})$ have zero surface divergence at any point of the membrane.

The total force $\boldsymbol{F}^{\text {tot }}$ is a sum of active and passive forces:

$$
\boldsymbol{F}^{t o t}(\boldsymbol{x})=F(\boldsymbol{x}) \boldsymbol{n}(\boldsymbol{x})+\boldsymbol{F}^{t e n s}(\boldsymbol{x}) .
$$

The Stokes equations for internal and external fluids can be solved following a procedure outlined, e.g., in [23]. Some technical details can be found in [22], while here we merely focus on the outcome.

The results

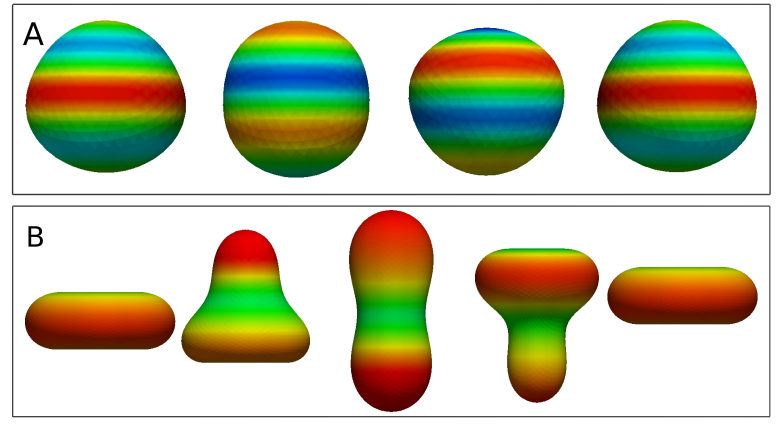

FIG. 1: (Color on-line) A: Shape transformation during a three-stroke cycle for fastest swimming. $l_{\max }=16$. The normed excess area $\Gamma=0.025$. Color code shows the distribution of active forces necessary to go to the next shape. B: Large deformation swimmer with $\Gamma=0.16$. Colors of the insets correspond to $F(\boldsymbol{x})$ (red outward, blue inward directed force).

Axisymmetric swimming This configuration is sufficient to expose the main results. Only harmonics with $m=0$ survive (below the subscript ' $m$ ' is omitted, i.e. $\rho_{l, 0} \equiv \rho_{l}$ and $F_{l, 0} \equiv F_{l}$ ). To the leading order, the evolution of the shape of the swimmer obeys [22]:

$$
\dot{\rho}_{l}(t)=\beta_{l}\left[F_{l}(t)-\frac{\zeta_{0}(t)}{r_{v}} \alpha_{l} \rho_{l}(t)\right], l>1,
$$

where $t$ is the time, $\dot{\rho}_{l}(t)=d \rho_{l}(t) / d t$ and the swimming velocity $v^{c}$ is found to be given by

$$
v^{c}=\dot{r}^{c}(t)=r_{v} \sum_{l=2}^{l_{\max }-1}\left[\mu_{l} \rho_{l+1}(t) \dot{\rho}_{l}(t)-\nu_{l} \rho_{l}(t) \dot{\rho}_{l+1}(t)\right] .
$$

The coefficients $\alpha_{l}, \beta_{l}, \mu_{l}$ and $\nu_{l}$ depend on $l$ and viscosity contrast $\lambda$ and are listed in [22].

The isotropic part of the Lagrange multiplier $\zeta_{0}$ is calculated by substituting (4) into the time derivative of fixed surface area condition yielding

$$
\sum_{l=2}^{l_{\max }} \frac{\alpha_{l} \rho_{l}^{2}}{2(2 l+1)}=\Gamma
$$

$\zeta_{0}$ is a nonlinear function of $\rho_{l}[22]$ so that Eq.(4) is nonlinear in $\rho$ (its explicit form is listed in [22]). This markedly differs from studies not imposing incompressibility where the evolution equations are linear [15]. Results (4) and (5) are our basic equations.

Swimming pattern In order to mimic the swimming strategies of actual living organisms, we allow for a rather restricted set of swimming patterns: we assume that the swimming can be represented as a series of elementary strokes, the intervals of time, during which the distribution of active normal forces $F(\boldsymbol{x})$ does not vary. If the 
amplitudes $F_{l}$ are constant in time, the solution of (4) relaxes to a steady-state value, the time derivatives $\dot{\rho}_{l}$ decrease to zero exponentially and the swimmer stops moving according to (5). It must then change the distribution of normal active forces, thus starting a new elementary stroke. In this study, we consider full elementary strokes, i.e., the strokes that last until the shape reaches saturation and the propulsion stops. The distance traveled during one full elementary stroke does not change if all force amplitudes $F_{l}$ are multiplied by the same number. Indeed, multiplying $F_{l}$ and $\zeta_{0}(t)$ by a constant $a$ and dividing the time $t$ by $a$ leaves Eqs. $(4,5,6)$ invariant. In other words, if the amplitude of active forces is doubled, the shape of the swimmer reaches the saturation twice faster, so that despite the fact that at the beginning of the stroke the velocity is doubled, it falls to zero twice faster, and the traveled distance remains unchanged. Thus, the distance during an elementary stroke is unambiguously defined by the shape of the swimmer at the beginning and at the end of the stroke.

Swimming strategy among a large manifold of possibilities Consider a stroke cycle, a sequence of full elementary strokes that returns the swimmer to its initial shape. If the swimmer exhibits a finite displacement during one stroke cycle, it can swim any distance by duplicating the strokes. The swimming velocity is equal to the displacement during one stroke cycle times the frequency of the stroke cycles. One could ask then: "What would be the fastest way to swim?" That is, which distributions of active normal forces during elementary strokes the swimmer should deploy in order to achieve the maximal displacement during one stroke cycle.

For analytical tractability, we set $l_{\max }=3$. This entails that only $\rho_{2}$ and $\rho_{3}$ are non zero (see (4)), but are linked by constraint (6) leaving us with only one degree of freedom. Interestingly enough, swimming can occur despite a single degree of freedom; this does not contradict the scallop theorem, as shown below. The constraint (6) describes an ellipse in the $\left(\rho_{2}, \rho_{3}\right)$ plane. The simplest non-trivial cycle of shape changes corresponds to a complete turn around the ellipse. The displacement after one cycle is obtained by time-integration of (5):

$$
\begin{aligned}
& \Delta r_{z}^{c}=\int_{0}^{T} \dot{r}_{z}^{c} d t=\int_{0}^{T}\left[\mu_{2} \rho_{3}(t) \dot{\rho}_{2}(t)-\nu_{2} \rho_{2}(t) \dot{\rho}_{3}(t)\right] d t \\
& =\oint \mu_{2} \rho_{3} d \rho_{2}-\nu_{2} \rho_{2} d \rho_{3}=\left(\mu_{2}+\nu_{2}\right) \int d \rho_{2} d \rho_{3}
\end{aligned}
$$

where $T$ is the length of the stroke cycle. The last integral in (7) is the area of the ellipse. From the equation of the ellipse (6) the area is determined, and (7) yields (by using expressions of $\mu_{l}$ and $\nu_{l}$ given in [22])

$$
\Delta r_{z}^{c}=\frac{3 \pi \Gamma}{\sqrt{14}} r_{v} \simeq 2.5 \Gamma r_{v}
$$

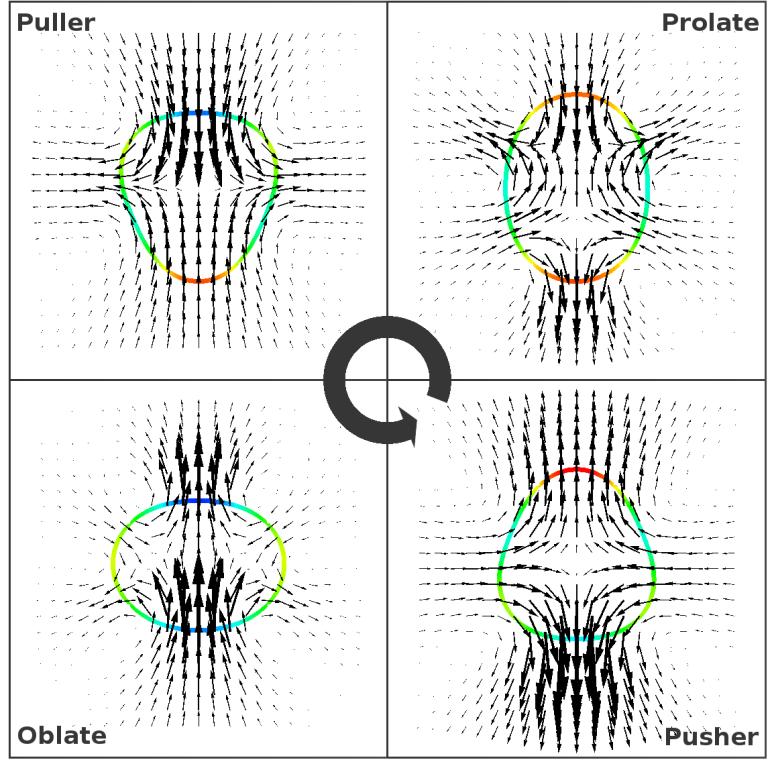

FIG. 2: (Color on-line) Numerical simulations of flow around a swimmer. Time flow is counter-clockwise. Symmetry axis of the swimmer is vertical. Color code shows the intensity of the active force $F$. Swimming direction points towards the top of the page. $\Gamma=0.0134$.

This result is independent of the force distribution (as well as of their temporal evolution), or any other parameter, but involves only a universal constant and geometrical quantities. If higher harmonics are taken into account $\left(l_{\max }>3\right)$, however, there exists a large manifold of swimming patterns. The question naturally arises about the selection of swimming pattern. Previous studies used dissipation [15-19] as a criterion for selection. Here we optimize the swimming speed instead. We propose that the elementary strokes can only be performed at a finite rate due to the time required for the adaptation of the internal biochemical machinery of the cell. Therefore we choose to maximize the distance traveled during one stroke cycle while keeping the number of elementary strokes per cycle fixed. In order to find the optimal swimming strategy in this sense, we fix the number of elementary strokes during one cycle and solve for optimal distributions of normal forces $F_{l}$ during each elementary stroke by numerical maximization of $\Delta r_{z}^{c}$. We have checked that the maximum displacement during a three-stroke cycle is not too much affected upon increasing the number of spherical harmonics $l_{\text {max }}$ : for $l_{\max }=4, \Delta r_{\max }^{c} / r_{v}=3.10 \Gamma$, for $l_{\max }=6$ or $l_{\max }=16$, $\Delta r_{\max }^{c} / r_{v}=3.24 \Gamma$. The fastest three-stroke cycle for $l_{\max }=16$ and $\lambda=1$ is shown in Fig. 1A. A striking feature of this snapshot is the absence of cusps or high-wavevector wrinkles observed in optimal strokes of swimmers with extensible membrane[15-18]. We attribute this difference to the effect of membrane inextensibility. 
Pusher or puller? So far two main distinct swimmers have been identified in literature: pusher and puller $[2,24]$. It is thus natural to ask whether the present swimmer belongs or not to any of these two categories. The four strokes of the cycle are identified as follows (Fig. 2) : (i) oblate-like shape (bottom left Fig. 2) with $\left(\rho_{2}<0, \rho_{3}=0\right)$, showing flow lines in the swimming direction pointing along swimming, (ii) forwardpointed stage $\left(\rho_{2}=0, \rho_{3}>0\right)$, (bottom right) where flow lines point outward along swimming direction (typical for pushers), (iii) prolate-like stage $\left(\rho_{2}>0, \rho_{3}=0\right)$, (top right) with flow lines in the swimming direction pointing against swimming, and (iv) a backward-pointed stage $\left(\rho_{2}=0, \rho_{3}<0\right)$, where flow lines point inward along swimming direction (typical for pullers). Overall, within a cycle of swimming, pulling and pushing coexist in a complex entangled state, thus the denomination EPP (entangled pusher-puller) is adopted. It is found that the puller and pusher phases perfectly cancel each other (time-average swimmer stresslet is exactly zero) in the small deformation regime and when $l_{\max }=3$. This ceases to be the case in general if $l_{\max }>3$.

Strong shape deformation Real cells, like Eutreptiella gymnastica or neutrophils, undergo ample shape deformation. In order to dispose of a quantitative information, we have performed a full numerical simulation accounting for large deformations by means of 3D boundary integral formulation. This is a formidable task even for passive particles (like red blood cells under flow), resolved only recently [25]. We set $\Gamma=0.16$. and apply active forces specified by two non-zero amplitudes (for illustration)

$$
F_{2}=2 \cos (\omega t), \quad F_{3}=-2 \sin (\omega t) .
$$

Despite this form, higher harmonics are excited in the shape of the swimmer. The swimming frequency is set to $\omega=0.05$ (the swimmer has sufficient time to adapt to the distribution of active forces). Swimming snapshots are shown in Fig.1B, resembling those of Eutreptiella gymnastica. Figure 3 shows the instantaneous velocity. We found for the simulation reported in Fig.1 that on average the swimmer behaves as a puller.

A systematic numerical analysis for various values of $\Gamma$ for a given force distribution is performed. Interestingly enough the propulsion distance over one swimming cycle follows almost a linear relationship with $\Gamma$

$$
\frac{\Delta r_{z}^{c}}{r_{v}} \sim c \Gamma, \quad c \simeq 2.4-2.7
$$

The prefactor is close to that in (8). The results are reported in Fig. 4. Result (10) can be understood from a heuristic argument. The active force $F$ creates a viscous tension, $F \sim \eta V / R_{A}$, where $R_{A}$ is a typical length scale of the cell protrusion, of order of $\sqrt{\Gamma}$. Time scale for a stroke is given (from dimensional analysis) by $\tau \sim(\eta / F) a$ where $a$ is a dimensionless quantity defining how fast

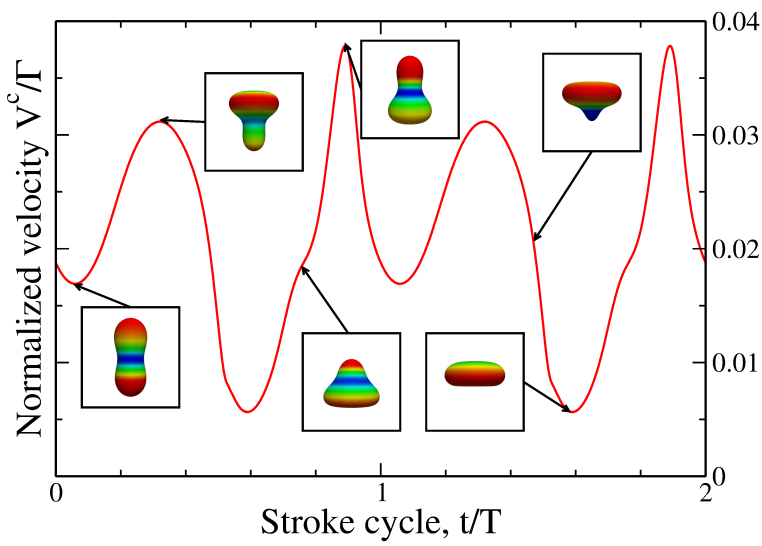

FIG. 3: (Color on-line) Swimming velocity as a function of time. $r_{0}=\eta=\lambda=1$. The insets show the instantaneous shapes of the swimmer. Colors of the insets correspond to $F(\boldsymbol{x})$ (red outward, blue inward directed force).

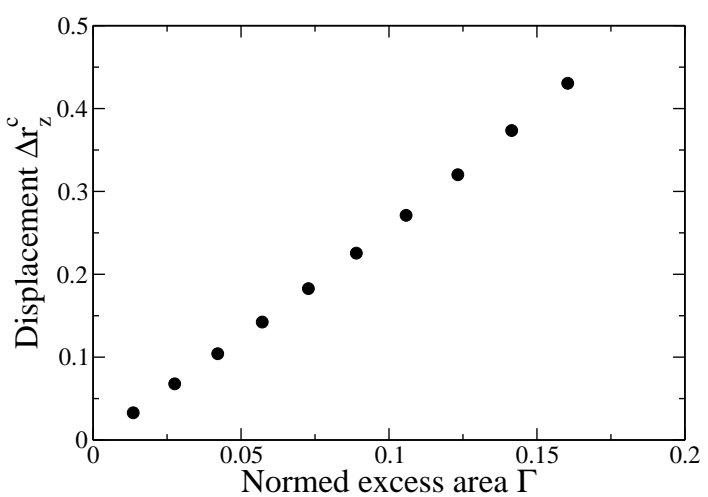

FIG. 4: Swimming propulsion distance over one cycle as a function of excess area $\Gamma$.

the shape achieves saturation. The more there is excess area the longest is $\tau$, and from the shape equation we have to leading order $a \sim \sqrt{\Gamma}$ (see [22]), so that the propulsion distance is $V \tau \sim \Gamma$. The law (10) is not devoid of experimental testability. For eutreptiella gymnastica with size of order $20 \mu \mathrm{m}$, with $\tau \sim 10 \mathrm{~s}$, we find a force of order $10 \mathrm{pN}$. The reduced area $\Gamma$ is of about 0.2 [20], and using (10), we find $\frac{\Delta r_{z}^{c}}{r_{v}} \sim 0.4$ and a speed of propulsion (by using the stroke frequency) of about $1 \mu \mathrm{m} / \mathrm{s}$. These are consistent with known data [20].

In conclusion, the peculiar motion of eutreptiella is recovered with a simple distribution of normal forces, and the propulsion distance is obtained in terms of geometrical quantities. Some euglenids change volume during swimming [20], whereas several animal cells seem to change area during spreading on substrate [26] (the situation during swimming is unclear). It is hoped to investigate this matter in a future work.

Acknowledgments.- We acknowledge fruitful discussion with Karin John. A.F. and C.M. gratefully acknowledge 
financial support from CNES and ESA. S.R. and P.P. are supported by ANR, project MICMACSWIM.

* Electronic address: alexandr.farutin@ujf-grenoble. fr

$\dagger$ Electronic address: chaouqi.misbah@ujf-grenoble.fr

[1] E. M. Purcell, Am. J. Phys. 45, 3 (1977), URL http: //dx.doi.org/10.1119/1.10903.

[2] E. Lauga and T. Powers, Reports on Progress in Physics 72, 096601 (2009)

[3] M. L. Ginger, N. Portman, and P. G. McKean, Nature Reviews Microbiology 6, 838 (2008).

[4] K. F. Jarrell and M. J. McBride, Nature Reviews Microbiology 6, 466 (2008).

[5] J. Gray and G. Hancock, Journal of Experimental Biology 32, 802 (1955).

[6] H. C. Berg, E. coli in Motion (Springer, 2004).

[7] T. Ishikawa and T. Pedley, J. Fluid. Mech. 588, 437 (2007).

[8] M. Garcia, S. Berti, P. Peyla, and S. Rafaï, Physical Review E 83, 035301 (2011).

[9] L. Turner, W. Ryu, and H. Berg, J. Bacteriol. 182, 2793 (2000).

[10] J. Throndsen, Norwegian Journal of Botany 16, 161 (1969).

[11] N. P. Barry and M. S. Bretscher, PNAS 107, 11376 (2010).

[12] A. J. Baea and E. Bodenschatz, PNAS 107, E165 (2010).

[13] T. Ohta and T. Ohkuma, Phys. Rev. Lett. 102, 154101 (2009), URL http://link.aps.org/doi/10. 1103/PhysRevLett.102.154101.

[14] T. Hiraiwa, K. Shitaraa, and T. Ohta, Soft Matter 7,
3083 (2011).

[15] A. Shapere and F. Wilczek, Phys. Rev. Lett. 58, 2051 (1987), URL http://link.aps.org/doi/10.1103/ PhysRevLett.58.2051.

[16] J. E. Avron, O. Gat, and O. Kenneth, Phys. Rev. Lett. 93, 186001 (2004), URL http://link.aps.org/doi/10. 1103/PhysRevLett.93.186001.

[17] F. Alouges, A. Desimone, and L. Heltai, Mathematical Models and Methods in Applied Sciences 21, 361 (2011), http://www.worldscientific.com/doi/pdf/10.1142/S02182025110 URL http://www.worldscientific.com/doi/abs/10. 1142/S0218202511005088.

[18] J. Lohéac, J.-F. Scheid, and M. Tucsnak, Acta Applicandae Mathematicae 123, 175 (2013), ISSN 0167-8019, URL http://dx.doi.org/10.1007/ s10440-012-9760-9.

[19] A. Vilfan, Phys. Rev. Lett. 109, 128105 (2012).

[20] M. Arroyo, L. Heltai, D. Millán, and A. DeSimone, Proceedings of the National Academy of Sciences 109, $17874 \quad$ (2012), http://www.pnas.org/content/109/44/17874.full.pdf+html, URL http://www.pnas.org/content/109/44/17874. abstract.

[21] A. Z. K. Yazdani and P. Bagchi, Phys. Rev. E 84, 026314 (2011).

[22] supplementary materials.

[23] G. Danker, T. Biben, T. Podgorski, C. Verdier, and C. Misbah, Phys. Rev. E 76, 041905 (2007), URL http: //link.aps.org/doi/10.1103/PhysRevE.76.041905.

[24] D. Saintillain and M. Shelley, C.R. Physique 14, 497 (2013).

[25] A. Farutin and C. Misbah, Phys. Rev. Lett. 109, 248106 (2012).

[26] M. Sheetz, J. Sable, and H. Dobereiner, Annu. Rev. Biophys. Biomol. Struct. 35, 417 (2006). 